\title{
Consistency Based Sensor Defect Detection
}

\author{
Jan-Friedrich Ehlenbröker, Uwe Mönks, Volker Lohweg \\ inIT - Institute Industrial IT, Langenbruch 6, 32657 Lemgo, Germany \\ jan.ehlenbroeker@hs-owl.de
}

\begin{abstract}
:
One general problem is the detection of sensor defects. Defective sensors can have several negative consequences, e. g., they will lead to machine failure when wear and tear of a machine is not detected sufficiently in advance. In this contribution we present a method to detect faulty sensors by calculating the consistency between sensor values. Background for this consistency-driven approach is a sensor fusion algorithm which combines sensors to attributes. These attributes are generally created based on local or thematical proximity. Therefore a consistency based approach is promising.
\end{abstract}

Key words: sensor defect, sensor fusion, reliability

\section{Introduction}

When using a sensor in an application it is generally assumed that it is operating correctly. But sensors can fail and do so during typical operation. This failure can be caused by wrongful handling, by wear and tear, or it can be a random failure. The failure may be a complete failure of the sensor, which is easily detectable, as the sensors stop delivering any data. Other defects are harder to detect.

When a sensor continuously delivers values, it is not necessarily directly detectable or decidable if the sensor measurements are valid or not. The sensor might produce values that deviate more from the true value than their given accuracy. These types of defects are problematic. An example is the application of sensors for condition monitoring purposes in manufacturing processes. Here, sensor defects can lead to a decrease in product quality or a reduction in the produced quantity of a given product. Depending on the use case of the sensor it is also possible that a sensor defect can have more severe consequences.

There are multiple possible ways to detect and handle sensor defects. One approach is the usage of intelligent sensors that execute selftests to detect the sensor performance and sensor defects. Depending on the type of sensor and the intended use, this may be a valid way to handle sensor defects. On the other hand, the self-testing capabilities are often limited to simple function tests. Additionally, intelligent sensors have higher acquisition costs compared to sensors without self-test abilities. Moreover, sensors with selftest abilities are not available for every use case, especially when applications impose special requirements, e.g. explosion protection.

A different approach is the usage of multiple sensors for monitoring of one and the same object or property. A simple way to further process the observations of multiple sensors is a threshold system. Every sensor observation is classified individually based on a threshold. These results are fed into a majority voting system to generate a global status [1]. Following such an approach can lower the impact of a sensor defect, as multiple nondefective sensors can overrule one defective sensor. Nevertheless, such systems are too simple to model a complex application sufficiently.

Hence, more adequate (but also more complex) data fusion algorithms are used for the processing of sensor observations and the generation of a system state. Such data fusion methods have been around for many years [2]. One of such data fusion algorithm is the multilayer attribute-based conflict-reducing observation (MACRO, [3, 4]) algorithm. It has shown good performance, especially in situations, where the fused data is conflicting [5].

Conflicting states are often caused by defective sensors. Therefore the ability of a data fusion system to handle conflicts is important. But while MACRO shows good performance concerning conflicts between sensors, there is no direct way for the fusion algorithm to detect defective sensors. For other sensor fusion approaches similar statements can be made: There a many approaches in the literature for the detection of process anomalies by using 
sensor fusion methods, including methods that handle or reduce conflict between sensor observations [2]. Some sensor fusion approaches incorporate sensor reliability values (e.g. [6], [7]). In these case the reliability is used as a discounting factor during the sensor fusion process. But almost none of these sensor fusion algorithms do use the sensor conflict or consistency measures as a method to predict or detect defective sensors. One exception is [8], in which a conflict-based detection method for defective sensors is proposed.

In this paper we propose a new method that uses the inherent multi-layer structure of MACRO with the help of consistency calculations between sensors for sensor defect detection.

The application, in which this approach of condition monitoring and sensor defect detection is demonstrated, is the research project itsowl-IGel [9]. One goal of IGel is the development of a condition monitoring and early warning system for hazardous material stores. Hazardous material stores are used to safely store materials like dangerous chemicals. Storage of these materials is regulated, based on the properties of the stored materials. For example the store must be fitted with a pan that collects chemicals in case of a leakage. Depending on the chemical, the hazardous material store must also be built to withstand fires.

This paper is separated into the following chapters: Approach, where we first give a general overview over the data fusion algorithm MACRO, followed by a more detailed look into the method for sensor defect detection. The experiments and results are given in the following chapter. The paper concludes with a chapter that gives an overview over the results and delivers an outlook on future work.

\section{Approach}

The approach section is divided into multiple parts: First a brief description of the used data fusion algorithm is presented. This section is followed by a background section where a preliminary for the following parts is given. Sections on the sensor consistency and reliability calculation conclude the approach.

\section{Multi-layer Attribute-based Conflict-reducing Observation (MACRO)}

For the fusion of several sensor signal input, we make use of the multi-layer attribute-based conflict-reducing observation (MACRO) approach. It has been shown that the application of this approach for hazardous material store monitoring is beneficial compared to usual installations [10]. MACRO's structure is depicted in Fig. 1 and the fusion is carried out as follows:

For the determination of a hazardous material store's global state, signals of itself as well as its environment (like oxygen concentration, temperature, humidity) are acquired by sensors (signal sources).

Features are extracted from the signals in the following signal conditioning step which may also include signal preprocessing procedures. Ensembles of conditioned signals are then grouped to so-called attributes representing certain properties or physical parts of the observed store, such as air quality, ventilation, air conditioning. During a first training phase under ensured good condition, the features acquired are learned and used for reference in the monitoring phase. The attributes are application-dependent and defined during the design process. Redundancies occurring by combining at least two information sources to one attribute are used beneficially for both (i) intercepting sensor faults and (ii) crosschecking the consistency of sensor values.

The latter is carried out implicitly by the psychologically inspired fuzzified balanced twolayer conflict solving ( $\mu$ BalTLCS) fusion approach [5]: $\mu$ BalTLCS creates one output signal per attribute from its input signals and assigns the attribute an importance measure which is the negated conflict between the sensors' individual opinions. Conflict occurs whenever information does not bear evidence for only one opinion/proposition, but also for another. This might either be due to actual failure in the process or system observed, or caused by one or some defective sensors. The latter case is the most severe one as wrong decisions might be derived if sensors were considered reliable, although they are not.

Subsequently, the fused attributes' opinions ( $\mu$ BalTLCS output signals) are aggregated on system level using the Implicative Importance Weighted Ordered Weighted Averaging (IIWOWA) operator [11] to reason about the entire store under supervision. It weights each attribute according to its previously determined importance, such that attributes with a high conflict have only small or no impact on the aggregation result.

Detailed information regarding MACRO can be found in $[4,5]$. 


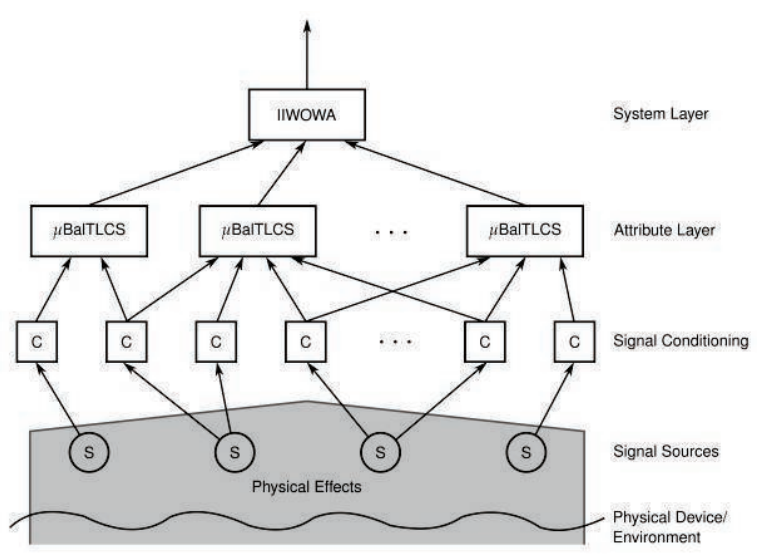

Fig. 1. Multi-layer attribute-based conflict-reducing observation system MACRO.

In this work we concentrate on MACRO's core fusion part $\mu$ BalTLCS on the attribute layer. Here, the sensor signals are fused initially and checked for consistency.

\section{Background}

Our approach takes the sensors that are combined to an attribute and calculates consistency values for every sensor. These consistency values are calculated based on the sensor values for a given point in time and are in turn used to generate a reliability value for every sensor. The proposed approach is based on parts of the sensor fusion algorithm proposed by Glock et al. [12].

Sensor observations are modeled as possibility distribution, a concept based on the possibility theory, introduced by Zadeh in 1978 [13]. A short definition and introduction of the used aspects of the possibility theory follows: Given $X$ is the universe of discourse, the variable $v$ represents an imprecisely known value of a feature $F$ inside $X$. The variable $v$ may for example be the temperature of an object or a distance measurement. We now model this feature as a fuzzy set $F$ with the characteristic membership function being $\mu_{F}(x)$. The only available knowledge about $v$ is that " $v$ is in $F$ ", with $F \subseteq X$. If this is true the possibility distribution is defined as follows [13]:

$$
\pi_{v}(x)=\mu_{F}(x), \forall x \in X .
$$

In case of the sensor $S$ the associated possibility distribution is $\pi_{v}^{i}(x)$.

More details on the possibility theory and the mathematical background of the following approach can be found in [12].

\section{Consistency Calculation}

Given an attribute $A$ that combines the $n$ sensors $S^{A}=\left\{S_{1}, S_{2}, \ldots, S_{i}, \ldots, S_{n}\right\}$, every sensor has a reliability value $\left(\left\{\rho_{1}, \rho_{2}, \ldots, \rho_{i}, \ldots, \rho_{n}\right\}\right)$. We now look for the largest set of sensors that form a consensus observation $T_{C}^{A}$ with $T^{A} \subseteq S^{A}$ for every attribute:

$$
T_{C}^{A}=\left\{T^{A} \mid h\left(T^{A}\right)>0\right\} .
$$

In Eq. (2), $h$ is the consistency index of subset $T^{A}$, as defined in Eq. (3):

$$
\begin{aligned}
& h\left(\pi_{v}^{1}(x), \pi_{v}^{2}(x)\right)= \\
& \quad \sup _{x \in X}\left[\min \left[\pi_{v}^{1}(x), \pi_{v}^{2}(x)\right]\right] .
\end{aligned}
$$

Eq. (2) is only valid for sensors with a reliability of $\rho_{i} \geq 0.5 \forall S_{i} \in T^{A}$. Sensors with a lower reliability are essentially delivering random results and are therefore neglected in Eq. (2).

To reduce the calculation costs for the consistency of every sensor, we defuzzify every sensor possibility distribution. Defuzzification is done by calculating the discrete center of gravity [14]:

$$
C\left(\pi_{v}^{i}\right)=\frac{\sum \pi_{v}^{i}(x) \cdot x}{\sum \pi_{v}^{i}(x)} .
$$

The consensus observation $T_{C}^{A}$ is limited by the minimum and the maximum center of gravities of all sensors of $T_{C}^{A}$, as described in Eq. (5) and Eq. (6):

$$
\begin{gathered}
c_{\min }=\min _{S_{i} \in T_{c}^{A}}\left[C\left(\pi_{v}^{i}\right)\right], \\
c_{\max }=\max _{S_{i} \in T_{c}^{A}}\left[C\left(\pi_{v}^{i}\right)\right] .
\end{gathered}
$$

The consistency of a sensor value is calculated based on the distance between the sensor observation and the consensus observation:

$$
\begin{aligned}
& \operatorname{Co}_{C}\left(\pi_{v}^{i}, T_{c}\right)= \\
& \left\{\begin{array}{cc}
1-\left(c_{\min }-C\left(\pi_{v}^{i}\right)\right), & C\left(\pi_{v}^{i}\right)<c_{\min }, \\
1-\left(C\left(\pi_{v}^{i}\right)-c_{\max }\right), & C\left(\pi_{v}^{i}\right)>c_{\max }, \\
1, & \text { otherwise. }
\end{array}\right.
\end{aligned}
$$

When $T_{C}^{A}$ is not unique, the consensus observation is chosen based on the highest average reliability of the subsets. If this leads to no unique result, the consensus observation is chosen based on the highest consistency. In case these steps do not lead to a unique consistency observation, the consistency measure is calculated based on the distance 
between the observations of sensor $S_{i}$ and every other sensor of attribute $A, \pi_{v}^{i}$ and $\pi_{v}{ }^{*}$ :

$$
\begin{aligned}
& C o_{A}\left(\pi_{v}^{i}, \pi_{v}^{*}\right)= \\
& \quad \max \left[1-\max \left[\boldsymbol{\rho}_{i}\right], \lambda_{\text {WAM }}\left(\boldsymbol{\rho}_{i}, \mathbf{d}_{i}\right)\right],
\end{aligned}
$$

$$
\begin{gathered}
\text { with } \pi_{v}^{*}=\left\{\pi_{v}^{j}\right\}_{j \neq i}, \boldsymbol{\rho}_{i}=\left(\rho_{j}\right)_{j \neq i}, \mathbf{d}_{i}=\left(d_{i, j}\right)_{j \neq i} \text { and } \\
d_{i, j}=1-\left|C\left(\pi_{v}^{i}\right)-C\left(\pi_{v}^{j}\right)\right| .
\end{gathered}
$$

In Eq. (8), the distances are weighted with the reliability of the corresponding sensor. The Weighted Arithmetic Mean (WAM) is used for this weighting operation. The maximum reliability of every other sensor is in addition used for the calculation of a lower bound of the consistency of sensor $S_{i}$. This results in a consistency of 1 , when all observations $\pi_{v}^{*}$ are unreliable.

Summarizing, the calculation of the consistency of sensor $S$ is calculated based on the distance to the consensus observation, if there is one, and based on the distance to all other sensors, otherwise (cf. Eq. (10)):

$$
\operatorname{Co}\left(\pi_{v}^{i}\right)=\left\{\begin{array}{l}
\operatorname{Co}_{C}\left(\pi_{v}^{i}, T_{C}^{A}\right),\left|T_{C}^{A}\right|>1 \\
\operatorname{Co}_{A}\left(\pi_{v}^{i}, \pi_{v}^{*}\right), \text { otherwise. }
\end{array}\right.
$$

As the consistency of one sensor can fluctuate between different moments in time, it is sensible to evaluate the sensor consistency over a longer time period. Hence, instead of using the consistency of a sensor directly, we further process the consistency. This processed consistency is in the following called reliability of the sensor.

An infinite impulse response filter [15] is used for the calculation of the reliability:

$$
q[k]=\omega \cdot u[k]+(1-\omega) \cdot q[k-1] .
$$

In Eq. (11), $q[k]$ is the output of the filter at the discrete point in time $k$. In addition, $q[k-1]$ denotes the output at the discrete point in time $k-1$, while $u[k]$ is the input value at time $k$. Factor $\omega$ is set to a value between 0 and 1 $(\omega \in[0,1])$.

The reliability $\rho_{i}^{A}$ of the sensor $S_{i}$ in attribute $A$ is the minimum value of the static reliability $r_{s}^{i}$ and the dynamic reliability $r_{d}^{i}$ :

$$
\rho_{i}^{A}[k]=\min \left[r_{s}^{i}[k], r_{d}^{i}[k]\right] \text {. }
$$

The static reliability is based on historical experiences with the sensor itself. It can be calculated based on the statistical failure rate of the sensor hardware or set based on expert knowledge. Eq. (13) shows the calculation of the dynamic reliability, which is based on the infinite impulse response filter, as given in Eq. (11):

$$
r_{d}^{i}[k]=\omega \cdot \operatorname{Co}\left(\pi_{v}^{i}\right)+(1-\omega) \cdot \rho_{i}[k-1] .
$$

For all points in time with $k<0$, the dynamic reliability is set to 1 :

$$
r_{d}^{i}[k]=1 \forall k<0 .
$$

The factor $\omega$ is a smoothing factor for the reliability. High values of $\omega$ will show changes more directly, while small values smooth the reliability and will show reliability changes more slowly. An overview of the influence of $\omega$ is shown in Fig. 2.

As one sensor is often used in multiple attributes, we use the mean value over all attributes to get the reliability at time $k$ :

$$
\rho_{i}[k]=\frac{1}{m} \sum_{n=1}^{m} \rho_{i}^{A_{n}}[k] .
$$

In Eq. (15), $\left\{A_{1}, \cdots, A_{m}\right\}$ are denoting the attributes where sensor $S_{i}$ is used, with $m$ being the number of attributes.

\section{Experiments and Results}

The use case is, as previously stated, a hazardous material store. In the IGel project we use different types of sensors:

- Temperature Sensors

- Infrared Temperature Sensors

- Smoke Detectors

- Differential Pressure Sensors

- Gas Detectors

- Leakage Detectors

- Humidity Sensors

- Power Sensors

31 sensors are positioned in total on the inside, the air ducts, and the outside of the hazardous material store. These sensors are combined to 20 attributes.

Our sensor observations are not acquired as possibility distributions. Therefore we treat every sensor observation as a uniform probability density function (PDF). The PDF is given by the sensor observation and the sensor accuracy. This PDF is then transformed into a possibility distribution with the help of a 
Truncated Triangular Probability-Possibility Transform [16].

As the previously described reliability calculation is still being implemented into the hazardous material demonstrator store, we use synthetic and simulated sensor data. A first example is given in Fig. 2. The smoothing effect of lower $\omega$ values is clearly visible.

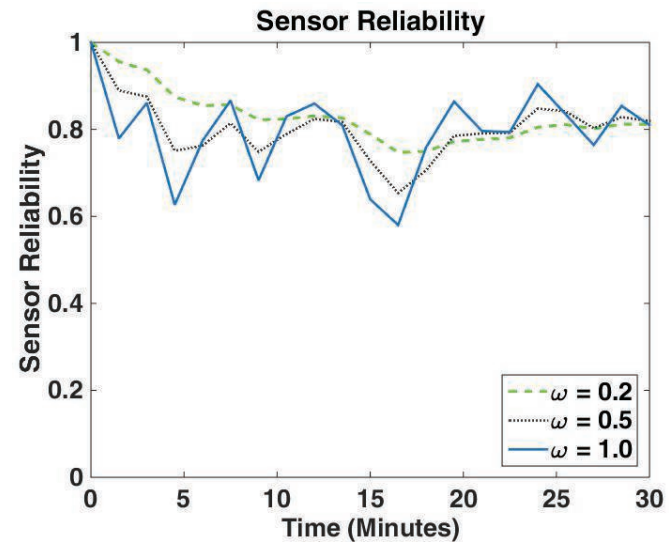

Fig. 2. Development of the reliability of one sensor with $\omega=0.2, \omega=0.5$ and $\omega=1.0$.

A more complex scenario is depicted in Fig. 3. The data used for this experiment has also been used in [10]. The sensor data simulated in [10] depicts the development of a smoldering fire. Smoldering fires release only a low amount of heat energy and smoke, compared to fully developed fires. Nevertheless they are dangerous in the context of a hazardous material store, especially since many of the stored chemicals are highly explosive. Smoldering fires can also develop into fully developed fires. During the simulation, only temperature sensors inside the store were influenced by the simulated smoldering fire.

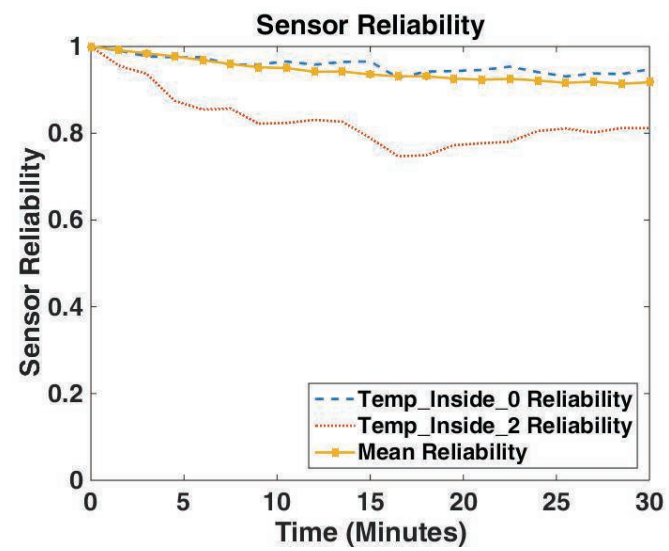

Fig. 3. The mean reliability over all sensors and the exemplary reliability of two sensors.

In Fig. 3 the reliability of two of the built in temperature sensors and the mean value over all sensors is depicted. The reliability of the Temp Inside 2 sensor decreases for about 10 minutes to a reliability value of about 0.8 . After that the reliability fluctuates around this value. The decreased reliability is the result of generally lowered consistency, as the sensors are distributed inside the storage unit. So while they overall measure the same process, they do not measure the exactly identical property.

The temperature sensor Temp_Inside_2, for example, measures the temperature above the smoldering fire at the upper left inside store. Due to its proximity to the heat source it is an outlier, compared to other temperature sensors. Therefore, it has been assigned a lower consistency and consequently lower reliability compared to other sensors.

The other temperature sensor shown is further away from the source of heat. It is therefore not as heavily influenced by the temperature change and its reliability values are slightly higher and comparable to the mean reliability.

The data used for Fig. 4 is identical to the one used in Fig. 3, with one change: In Fig. 4 a defect of sensor Temp_Inside_0 is simulated. This is carried out by setting the sensor measurement to a low value, opposite to the generally increasing temperatures.

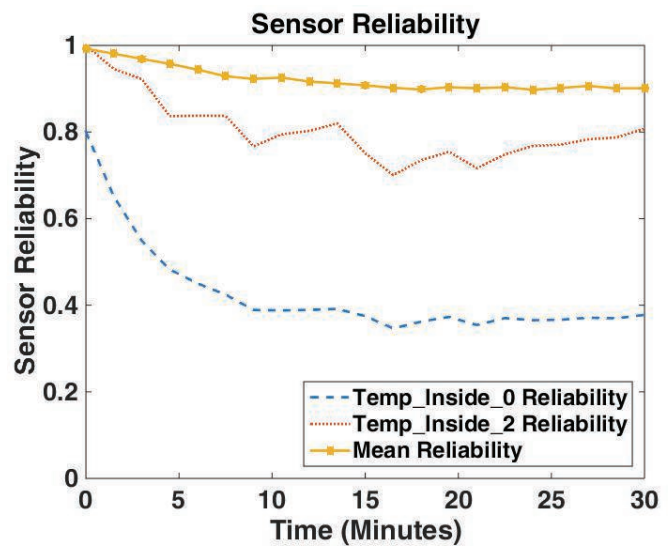

Fig. 4. The mean reliability and the reliability of two sensors. Sensor Temp_Inside_0 has a defect.

The defect of the sensor Temp_Inside_0 is clearly visible and detectable in Fig. 4. Especially if one looks at the distance between the reliability of sensor Temp_Inside_0 0 and the mean reliability over all sensors. In Fig. 4, a change of the reliability of sensor Temp_Inside_2 is also visible, compared to the results in Fig. 3. This is due to the calculation of the reliability: As the sensor values of sensor Temp_Inside_0 change, the reliability of other sensors may change, too.

\section{Conclusion and Outlook}

This paper presents a way to generate a consistency based reliability value for sensors. The approach has been embedded into the 
sensor fusion model MACRO. It has been demonstrated that this is a valid approach to detect sensor defects.

In the future, a more detailed look onto more complex scenarios might be of interest. The defect of multiple sensors should be researched, for example.

\section{Acknowledgement}

This work was partly funded by the German Federal Ministry of Education and Research (BMBF) within the Leading-Edge Cluster "Intelligent Technical Systems OstWestfalenLippe" (it's OWL).

\section{References}

[1] E. Alpaydin, Introduction to Machine Learning, 2nd ed. Cambridge, Mass.: MIT Press (2010), ISBN 026201243X.

[2] B. Khaleghi, A. Khamis, F. O. Karray, and S. N. Razavi, "Multisensor data fusion: A review of the state-of-the-art," Information Fusion, vol. 14, no. 1, pp. 28-44 (2011); doi: 10.1016/j.inffus.2011.08.001

[3] U. Mönks and V. Lohweg, "Fast Evidencebased Information Fusion," in 4th Int. Workshop on Cognitive Information Processing (CIP 2014): IEEE, pp. 1-6 (2014); doi: 10.1109/CIP.2014.6844508

[4] _ "Machine Conditioning by Importance Controlled Information Fusion," in 18th IEEE Int. Conf. on Emerging Technologies and Factory Automation (ETFA 2013): IEEE, pp. 1-8 (2013); doi: 10.1109/ETFA.2013.6647984

[5] U. Mönks, K. Voth, and V. Lohweg, "An Extended Perspective on Evidential Aggregation Rules in Machine Condition Monitoring," in 3rd Int. Workshop on Cognitive Information Processing (CIP 2012): IEEE, pp. 1-6 (2012); doi: 10.1109/CIP.2012.6232905

[6] Z. Elouedi, K. Mellouli, and P. Smets, "Assessing Sensor Reliability for Multisensor Data Fusion Within the Transferable Belief Model," IEEE Trans. Syst, Man, Cybern. B, vol. 34, no. 1, pp. 782-787 (2004); doi: 10.1109/TSMCB.2003.817056

[7] A. Martin, A.-L. Jousselme, and C. Osswald, "Conflict measure for the discounting operation on belief functions," in Information Fusion, 2008 11th International Conference on, pp. 1-8 (2008)

[8] V. Ricquebourg, L. Delahoche, B. Marhic, M. Delafosse, A. M. Jolly-Desodt, and D. Menga, "Anomalies recognition in a context aware architecture based on TBM approach," in Information Fusion, 2008 11th International Conference on, pp. 1-8 (2008)

[9] itsowl-IGel: Intelligentes Frühwarnsystem für Gefahrstofflager (Intelligent early warning system for hazardous material storage areas). Research Project sponsored by the Federal Ministry of Education and Research. Available: http://www.itsowl.com/projects/innovation- projects/details/intelligent-early-warningsystem-for-hazardous-material-storageareas/. (2015, Feb. 11),

[10] J.-F. Ehlenbröker, U. Mönks, D. Wesemann, and V. Lohweg, "Condition Monitoring for Hazardous Material Storage," in 19th IEEE Int. Conf. on Emerging Technologies and Factory Automation (ETFA 2014) (2014); doi: 10.1109/ETFA.2014.7005264

[11] H. L. Larsen, "Importance weighted OWA aggregation of multicriteria queries," in 18th Int. Conf. of the North American Fuzzy Information Processing Society (NAFIPS 1999), pp. 740-744 (1999); doi: 10.1109/NAFIPS.1999.781792

[12] S. Glock, K. Voth, J. Schaede, and V. Lohweg, "A Framework for Possibilistic Multisource Data Fusion with Monitoring of Sensor Reliability," in World Conference on Soft Computing (2011)

[13] L. A. Zadeh, "Fuzzy sets as a basis for a theory of possibility," Fuzzy sets and systems, vol. 1, pp. 3-28; http://www.mathuncertainty.org/Zadeh_1978_1999_Theory_of Possibility.pdf (1978)

[14] G. J. Klir and B. Yuan, Fuzzy Sets and Fuzzy Logic: Theory and Applications. New Jersey: Pentice Hall (1995), ISBN 9780131011717.

[15] U. Meyer-Baese, Digital signal processing with field programmable gate arrays, 3rd ed. Berlin, New York: Springer (2007), ISBN 3540726128.

[16] G. Mauris, L. Berrah, L. Foulloy, and A. Haurat, "Fuzzy handling of measurement errors in instrumentation," Instrumentation and Measurement, IEEE Transactions on, vol. 49 , no. 1 , pp. 89-93 (2000); doi: $10.1109 / 19.836316$ 